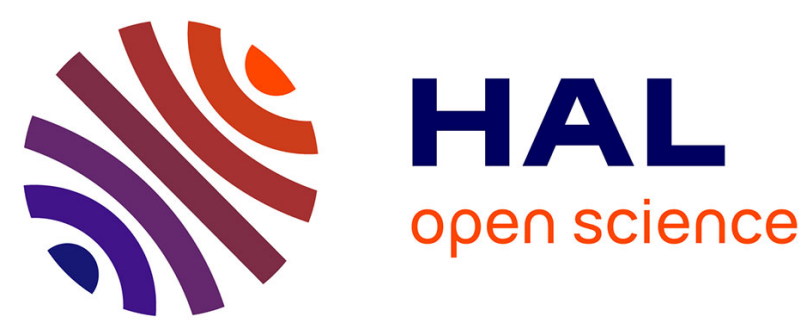

\title{
Effet de la composition chimique de la solution interstitielle de bétons jeunes sur la passivation d' un acier doux.
}

Sara Chakri, Pierre David, Isabelle Frateur, A. Galtayries, Philippe Marcus, Eliane Sutter, Bernard Tribollet, Vincent Vivier, S. Zanna

\section{To cite this version:}

Sara Chakri, Pierre David, Isabelle Frateur, A. Galtayries, Philippe Marcus, et al.. Effet de la composition chimique de la solution interstitielle de bétons jeunes sur la passivation d ' un acier doux. : Comportement à la corrosion d'un acier doux en environnement cimentaire. . Matériaux \& Techniques, 2015, Interactions microorganismes - matériaux de construction / Microorganisms-building materials interactions, 103 (2), pp.209. 10.1051/mattech/2015016 . hal-01179441

\section{HAL Id: hal-01179441 \\ https://hal.sorbonne-universite.fr/hal-01179441}

Submitted on 22 Jul 2015

HAL is a multi-disciplinary open access archive for the deposit and dissemination of scientific research documents, whether they are published or not. The documents may come from teaching and research institutions in France or abroad, or from public or private research centers.
L'archive ouverte pluridisciplinaire HAL, est destinée au dépôt et à la diffusion de documents scientifiques de niveau recherche, publiés ou non, émanant des établissements d'enseignement et de recherche français ou étrangers, des laboratoires publics ou privés. 


\title{
Effet de la composition chimique de la solution interstitielle de bétons jeunes sur la passivation d'un acier doux
}

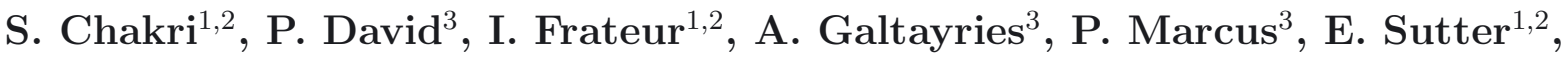 \\ B. Tribollet ${ }^{1,2}$, V. Vivier ${ }^{1,2}$ et S. Zanna ${ }^{3}$
}

Reçu le 16 janvier 2015, accepté le 9 avril 2015

Résumé - L'alcalinité de la solution interstitielle contenue dans les pores du béton engendre la formation d'une couche d'oxyde protectrice à la surface des armatures métalliques. L'objectif de ce travail est d'évaluer l'influence de la composition chimique de la solution interstitielle de bétons jeunes sur la qualité du film d'oxyde formé sur un acier doux C15. Pour ce faire, des mesures électrochimiques sont réalisées à pH 13, dans différentes solutions interstitielles synthétiques (CEM I, II et III), ainsi que dans une solution de référence $\mathrm{NaOH}$ 0,1 $\mathrm{M}$ et sont combinées à des analyses de surface par XPS. Les courbes de polarisation anodiques montrent l'intérêt des mesures stationnaires. Les diagrammes d'impédance électrochimique, tracés au potentiel de corrosion et à potentiels anodiques, mettent en évidence la présence d'une couche passive protectrice sur la surface d'acier, dont l'épaisseur est de l'ordre de 2,5 à $3 \mathrm{~nm}$, en bon accord avec celle estimée par XPS. Le pH n'est pas le seul paramètre déterminant pour prédire le comportement à la corrosion de l'acier. Ainsi, plus la concentration en sulfate en solution est importante, plus la densité de courant passif stationnaire est élevée et moins la solution est passivante. Par ailleurs, la présence de $\mathrm{Fe}^{2+}$ à la surface du matériau pourrait en partie expliquer le fort pouvoir passivant de la solution CEM III.

Mots clés : Béton armé / solution interstitielle / couche d'oxyde / courbes de polarisation / impédance électrochimique / XPS

\begin{abstract}
Effect of the chemical composition of fresh concrete pore solutions on the passivation of mild steel. The passivity of reinforcing steel in concrete is generally attributed to the formation of a protective oxide layer on the steel surface due to the high alkalinity of the surrounding pore solution. The objective of this work was to study the influence of the chemical composition of fresh concrete pore solutions on the electrochemical behaviour and the surface chemical composition of C15 mild steel, used for reinforcement of building concrete. For that purpose, electrochemical measurements were performed at $\mathrm{pH} 13$ in various simulated concrete pore solutions (CEM I, II and III), as well as in the $0.1 \mathrm{M} \mathrm{NaOH}$ reference solution, and were combined to surface analysis by XPS. The anodic polarization curves show the importance of steady-state measurements. The electrochemical impedance diagrams, plotted at the corrosion potential and at anodic potentials, show the presence of a protective passive layer on the steel surface with a thickness of about $2.5-3 \mathrm{~nm}$, in good agreement with XPS results. The $\mathrm{pH}$ is not the only determining parameter for predicting the corrosion behaviour of steel in concrete environments. Thus, the steady-state passive current density increases and the oxide layer becomes less protective with increasing sulfate concentration in solution. Furthermore, the strong protective nature of the passive layer in CEM III solution could be correlated to the presence of $\mathrm{Fe}^{2+}$ on the steel surface.
\end{abstract}

Key words: Reinforced concrete / pore solution / oxide layer / polarization curves / EIS / XPS

\footnotetext{
1 CNRS, UMR 8235, Laboratoire Interfaces et Systèmes Electrochimiques, France chakri.sara@gmail.com

2 Sorbonne Universités, UPMC Univ Paris 06, UMR 8235, LISE, 75005 Paris, France

3 CNRS-Chimie ParisTech, UMR 8247, Institut de Recherche de Chimie Paris, Équipe Physico-Chimie des Surfaces, 75005 Paris, France
} 


\section{Introduction}

Le béton fournit à l'armature métallique, habituellement en acier doux, une protection à la fois physique et chimique. La protection physique est assurée par la densité et la relative imperméabilité de sa structure. La protection chimique, quant à elle provient de l'alcalinité très élevée $(\mathrm{pH}>13,5)$ de la solution interstitielle contenue dans les pores du béton [1]. Un tel pH contribue à la formation et au maintien d'une couche passive, adhérente et stable, sur la surface de l'acier [2]. Le film passif ainsi formé peut être composé de $\mathrm{Ca}(\mathrm{OH})_{2}$ et de $\mathrm{Fe}(\mathrm{OH})_{2}$ ou $\mathrm{FeOOH}[3]$. Certains auteurs ont montré la présence d'une couche d'oxyde composée de $\mathrm{Fe}_{2} \mathrm{O}_{3}$ et de $\mathrm{Fe}_{3} \mathrm{O}_{4}$, dont l'épaisseur augmente progressivement avec le temps d'immersion [4]. Une structure spinelle $\alpha-\mathrm{Fe}_{3} \mathrm{O}_{4}-\gamma-\mathrm{Fe}_{2} \mathrm{O}_{3}$ a également été mise en évidence par d'autres auteurs $[4,5]$. Selon Gunay et al., le degré d'oxydation du fer présent dans la couche d'oxyde varie dans l'épaisseur de cette dernière : la couche interne est ainsi formée d'oxyde de $\mathrm{Fe}^{2+}$ protecteur, tandis que la couche externe est riche en oxyde et hydroxyde de $\mathrm{Fe}^{3+}$ moins protecteurs [6]. L'infiltration des ions chlorure et/ou la carbonatation du béton sous l'effet du dioxyde de carbone présent dans l'air, provoquant une diminution du $\mathrm{pH}$ jusqu'aux alentours de 9 , conduit à la rupture du film passif et à l'initiation de la corrosion par piqûres [7]. De telles conditions peuvent induire une détérioration partielle voire complète de la structure en béton.

Une solution saturée en hydroxyde de calcium est habituellement utilisée comme solution synthétique représentative de la solution interstitielle des bétons (CPS pour « concrete pore solution » en anglais) afin d'étudier le comportement électrochimique des armatures métalliques [8-10]. Cependant, la CPS contient également d'autres cations tels que $\mathrm{Ca}^{2+}, \mathrm{Na}^{+}$et $\mathrm{K}^{+}$et des anions tels que $\mathrm{OH}^{-}$et $\mathrm{SO}_{4}^{2-}$. Les ions sodium et potassium proviennent des oxydes alcalins, tels que $\mathrm{Na}_{2} \mathrm{O}$ et $\mathrm{K}_{2} \mathrm{O}$, présents dans le ciment. Quant aux ions sulfates, ils peuvent provenir du gypse $\left(\mathrm{CaSO}_{4} \cdot \mathrm{H}_{2} \mathrm{O}\right)$ ajouté lors de la fabrication du ciment, d'agents de contamination ou encore de l'eau utilisée lors du gâchage [11]. Il a ainsi été montré que la qualité du film d'oxyde formé à la surface de l'acier dépend, outre du pH et de la température, de la composition chimique de la solution et particulièrement de la présence d'ions sulfate [11].

L'objectif de cette étude est d'évaluer l'influence de la composition chimique de la solution interstitielle de bétons jeunes (quelques minutes après gâchage du ciment) sur le caractère protecteur du film d'oxyde formé à la surface d'un acier doux C15 généralement employé comme matériau constitutif des armatures métalliques dans les bétons de construction. Pour ce faire, des mesures électrochimiques au potentiel libre de corrosion $E_{c o r r}$ et dans le domaine anodique (suivi de $E_{c o r r}$ en fonction du temps d'immersion, tracé de courbes de polarisation et analyse par spectroscopie d'impédance électrochimique) sont combinées à des analyses de surface par spectroscopie de photoélectrons induits par rayons X (XPS). Les résultats obtenus dans différentes solutions interstitielles synthétiques sont comparés à ceux obtenus dans une solution de référence $\mathrm{NaOH} \mathrm{0,1} \mathrm{M} \mathrm{(pH} \mathrm{13)} \mathrm{simulant} \mathrm{l'environ-}$ nement basique des armatures métalliques et permettant de s'affranchir des autres ions entrant dans la composition des CPS.

\section{Matériaux et méthodes}

\subsection{Matériau métallique}

Le matériau étudié est un acier doux C15, généralement employé comme matériau constitutif des armatures métalliques dans les bétons de construction, selon la Norme EN 10277-2. Deux types d'échantillons sont utilisés : des électrodes à disque tournant (EDT) pour les mesures électrochimiques et des pastilles amovibles pour les analyses de surface. Dans les deux cas, la surface d'acier au contact de la solution est la section circulaire d'un cylindre de $0,8 \mathrm{~cm}$ de diamètre (surface géométrique égale à $\left.0,5 \mathrm{~cm}^{2}\right)$.

Pour les mesures électrochimiques, la surface latérale du cylindre est recouverte de peinture cataphorétique isolante avant d'être enrobée dans une résine époxy. Avant chaque expérience, l'EDT est polie au papier SiC de grain 1200 , nettoyée aux ultra-sons pendant une minute dans l'éthanol puis dans l'eau distillée et enfin séchée à l'argon.

Pour les analyses de surface, la surface latérale du cylindre est uniquement recouverte de peinture cataphorétique (pas d'enrobage dans la résine époxy). Avant chaque expérience, les échantillons sont polis au papier $\mathrm{SiC}$ de grain 1200, puis aux pâtes diamantées de $6 \mu \mathrm{m}$, $3 \mu \mathrm{m}$ et $1 \mu \mathrm{m}$, les étapes ultérieures de nettoyage et de séchage étant les mêmes que pour les EDT.

\section{2 Électrolytes}

Afin de préparer les solutions synthétiques représentatives de la solution interstitielle de bétons jeunes, différents ciments portland (CEM I 52,5 N, CEM II/B-S 42,5 N et CEM III/A 42,5 N-LH avec $61 \%$ en masse de laitier) sont gâchés avec un rapport eau sur ciment $\mathrm{E} / \mathrm{C}=0,45$ à l'aide d'un malaxeur IKA Eurostar 20 avec pale défloculeuse. Environ 1 min $30 \mathrm{~s}$ après gâchage, le mélange obtenu est filtré à l'aide d'une pompe à palettes, en utilisant deux filtres de 11 et $20 \mu \mathrm{m}$, puis à l'aide d'une seringue filtrante à 1 et $0,2 \mu \mathrm{m}$. Ce temps de filtration de $1 \mathrm{~min} 30 \mathrm{~s}$ est justifié par le fait que les concentrations élémentaires, en particulier celles en calcium et en soufre (sulfate), de la solution interstitielle ne varient pas pendant les 6 premières heures après gâchage [12]. Étant donné que les armatures métalliques sont mises en contact avec le béton $1 \mathrm{~h}$ après gâchage en moyenne, les filtrats de ciment obtenus dans ce travail peuvent être considérés comme représentatifs de la solution interstitielle réelle de bétons jeunes. Les solutions ainsi filtrées sont analysées afin de remonter à la composition en ions majoritaires. 
Tableau 1. pH et concentrations des ions majoritaires dosés dans les trois filtrats de ciment. $\mathrm{E} / \mathrm{C}=0,45$; filtration environ 1 min $30 \mathrm{~s}$ min après gâchage.

Table 1. $p H$ and concentrations of major ions measured in the three filtered cement solutions. $W / C=0.45 ;$ filtration about 1 min $30 \mathrm{~s}$ after mixing.

\begin{tabular}{cccccc}
\hline Ciment & $\mathrm{pH}$ & {$\left[\mathrm{Ca}^{2+}\right](\mathrm{mM})$} & {$\left[\mathrm{Na}^{+}\right](\mathrm{mM})$} & {$\left[\mathrm{K}^{+}\right](\mathrm{mM})$} & {$\left[\mathrm{SO}_{4}^{2-}\right](\mathrm{mM})$} \\
\hline CEM I & 13,6 & 16 & 88 & 350 & 190 \\
CEM II & 13,5 & 15 & 60 & 220 & 120 \\
CEM III & 13,2 & 19 & 37 & 190 & 80 \\
\hline
\end{tabular}

Les ions alcalins (sodium et potassium) sont dosés par spectrométrie d'absorption atomique. La concentration en sulfate est déterminée par ajout progressif d'une solution de nitrate de baryum et suivi conductimétrique de la solution en fonction du volume de $\mathrm{Ba}\left(\mathrm{NO}_{3}\right)_{2}$ ajouté.

Les ions calcium, sont dosés par potentiométrie, à l'aide d'une électrode sélective aux ions $\mathrm{Ca}^{2+}$. Les compositions des trois filtrats de ciment sont présentées dans le Tableau 1. Par ailleurs, le $\mathrm{pH}$ de ces 3 solutions est mesuré; les valeurs figurent également dans le Tableau 1.

À partir de ces compositions, des solutions synthétiques sont préparées avec les mêmes concentrations en ions majoritaires; ces solutions sont nommées par la suite CEM I, CEM II et CEM III. Avant chaque expérience, le $\mathrm{pH}$ de la solution d'étude est ajusté à 13 (valeur minimale de $\mathrm{pH}$ obtenue pour le filtrat de ciment CEM III), afin de s'affranchir de l'effet de différences éventuelles de $\mathrm{pH}$ entre les solutions. À la fin de chaque expérience, le $\mathrm{pH}$ est mesuré et sa valeur est toujours supérieure ou égale à 12,5. Par conséquent, cette faible variation de $\mathrm{pH}$ due à la carbonatation de la solution est supposée ne pas avoir d'effet notoire sur le comportement à la corrosion de l'acier.

Le comportement électrochimique de l'acier au carbone $\mathrm{C} 15$ a été également étudié dans une solution de référence $\mathrm{NaOH} 0,1 \mathrm{M}$ à $\mathrm{pH}=13$.

\subsection{Mesures électrochimiques}

La cellule électrochimique utilisée lors de ce travail était une cellule classique à trois électrodes, contenant environ $150 \mathrm{~mL}$ de solution. L'électrode de travail était l'EDT d'acier, la contre électrode une grille de platine de grande surface et l'électrode de référence une électrode au sulfate mercureux saturé (ESS; $\mathrm{Hg} / \mathrm{Hg}_{2} \mathrm{SO}_{4} / \mathrm{K}_{2} \mathrm{SO}_{4}$ saturé). Les expériences sont réalisées à température ambiante. Etant donné que le comportement électrochimique de l'acier dans le domaine anodique (passivation du matériau) ne dépend pas du transport de matière, les résultats présentés dans ce travail ont été obtenus en conditions statiques i.e. avec une vitesse de rotation de l'EDT égale à $0 \mathrm{tr} / \mathrm{min}$.

Les mesures électrochimiques sont réalisées à l'aide d'un potentiostat/galvanostat GAMRY Reference 600. Les diagrammes d'impédance électrochimique sont tracés dans un domaine de fréquence compris entre $10^{5} \mathrm{~Hz}$ et $10^{-2} \mathrm{~Hz}$, avec 7 points par décade et une amplitude de $10 \mathrm{mV}$ (rms).

\subsection{Analyses de surface}

Après immersion au potentiel de corrosion, les échantillons d'acier sont rincés avec la solution d'étude, puis séchés à l'argon avant introduction dans le spectromètre XPS (ESCALAB 250 ; Thermo Electron Corporation). Les analyses XPS sont réalisées en utilisant une source de rayons $\mathrm{X} \mathrm{Al} \mathrm{K} \mathrm{K}_{\alpha}$ monochromatique (1486,6 eV). La taille de la zone analysée est de $500 \mu \mathrm{m}$. En plus du spectre général (énergie de passage de $100 \mathrm{eV}$ ), les niveaux de cœur suivants sont systématiquement enregistrés à haute résolution (énergie de passage de $20 \mathrm{eV}$ ) : $\mathrm{Fe} 2 p, \mathrm{O} 1 s, \mathrm{C} 1 s, \mathrm{Na} 1 s, \mathrm{Ca} 2 p, \mathrm{~K} 2 p$ et $\mathrm{S} 2 p$. Toutes les analyses sont effectuées avec un angle d'émergence de $90^{\circ}$ (angle entre la surface et la direction dans laquelle les électrons sont analysés). Les données sont traitées à l'aide du logiciel Avantage (Thermo Electron Corporation), en utilisant une ligne de base de type Shirley et une forme de pics gaussienne/lorentzienne combinée. Les énergies de liaison sont calibrées par rapport au carbone : la liaison carbonée de type $\mathrm{C}-\mathrm{C}$ et $\mathrm{C}-\mathrm{H}$ a une énergie de liaison fixée à $285,0 \mathrm{eV}$.

\section{Résultats et discussion}

\subsection{Solution de référence $\mathrm{NaOH} 0,1 \mathrm{M}$}

\subsubsection{Durée de maintien à $E_{\text {corr }}$ nécessaire à l'obtention d'un état stationnaire}

Le potentiel libre ou potentiel de corrosion $E_{\text {corr }}$ de l'acier doux C15 a d'abord été suivi pendant les 100 premières heures d'immersion dans la solution $\mathrm{NaOH}$ 0,1 M afin de déterminer le temps nécessaire à la stabilisation du système (Fig. 1). Le potentiel de corrosion augmente considérablement pendant les premières heures d'immersion et atteint un maximum à $~ 80 \mathrm{~h}$. Après $100 \mathrm{~h}$, la valeur de $E_{c o r r}$ n'est toujours pas stationnaire et sa valeur est de l'ordre de $-0,55 \mathrm{~V} / \mathrm{ESS}$. Ces résultats montrent que la couche d'oxyde formée sur la surface de l'acier continue d'évoluer en composition, en structure ou en épaisseur après $100 \mathrm{~h}$ d'immersion.

Des diagrammes d'impédance sont tracés à $E_{c o r r}$ toutes les $8 \mathrm{~h}$ pendant les 100 premières heures d'immersion de l'électrode (Fig. 2). Tous les diagrammes de Nyquist présentent la même allure et se composent d'une boucle capacitive de large diamètre (de l'ordre de plusieurs centaines de $\mathrm{k} \Omega . \mathrm{cm}^{2}$ ), illustrant la présence d'une 


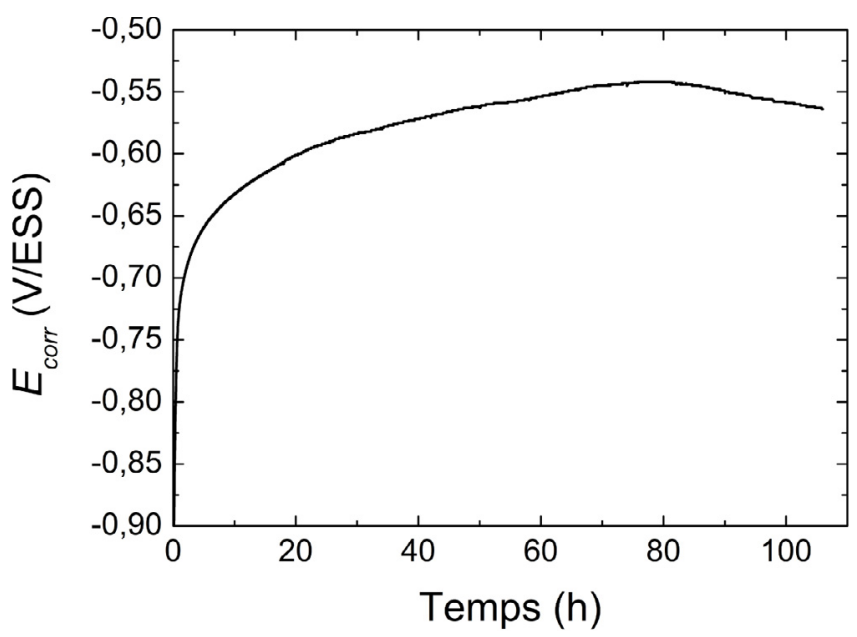

Fig. 1. Évolution du potentiel de corrosion $E_{\text {corr }}$ de l'acier C15 en fonction du temps d'immersion en solution $\mathrm{NaOH}$ $0,1 \mathrm{M}$.

Fig. 1. C15 mild steel corrosion potential $E_{\text {corr }}$ as a function of immersion time in $0.1 \mathrm{M} \mathrm{NaOH}$ solution.

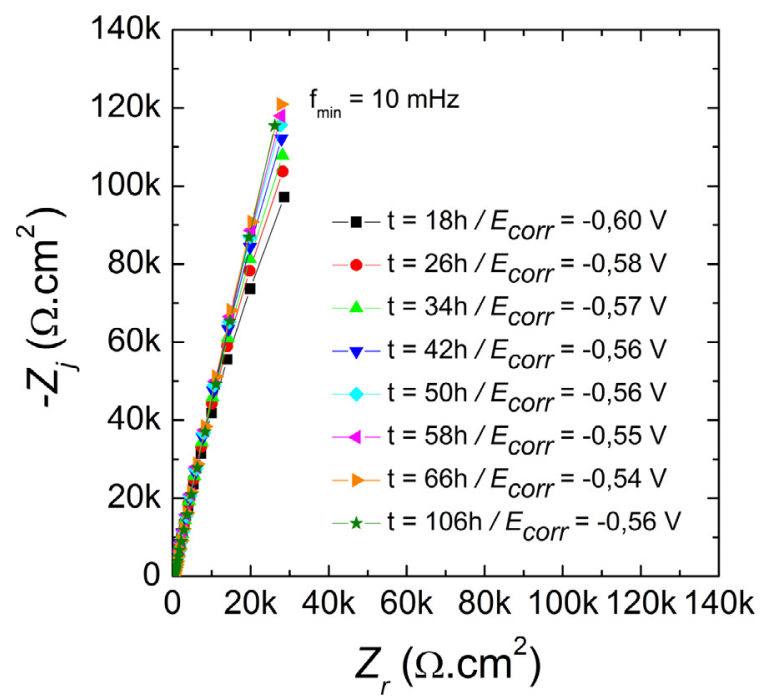

Fig. 2. Diagrammes d'impédance en représentation de $\mathrm{Ny}$ quist de l'acier $\mathrm{C} 15$ en solution $\mathrm{NaOH} 0,1 \mathrm{M}$, tracés à $E_{\text {corr }}$ à différents temps d'immersion. Nombre de points par décade : 7 .

Fig. 2. Impedance diagrams in the complex plane (Nyquist plots) of C15 mild steel in $0.1 \mathrm{M} \mathrm{NaOH}$ solution, plotted at $E_{\text {corr }}$ at different immersion times. 7 points per decade.

couche d'oxyde protectrice, de type couche passive, à la surface de l'acier. L'augmentation du diamètre de boucle avec le temps d'immersion, déjà observée par Carnot en milieu filtrat de ciment [13], montre que cette couche d'oxyde devient plus protectrice du fait de l'évolution de son épaisseur (augmentation selon Sagoe-Crentsil et Glasser [4]), de sa composition chimique (augmentation $\mathrm{du}$ rapport $\mathrm{Fe}^{2+} / \mathrm{Fe}^{3+}$ selon Ghods et al. [11]) et de sa structure.

À partir de ces diagrammes, on peut estimer le temps nécessaire à l'obtention d'un état quasi-stationnaire : il est égal au temps à partir duquel le diamètre de boucle,

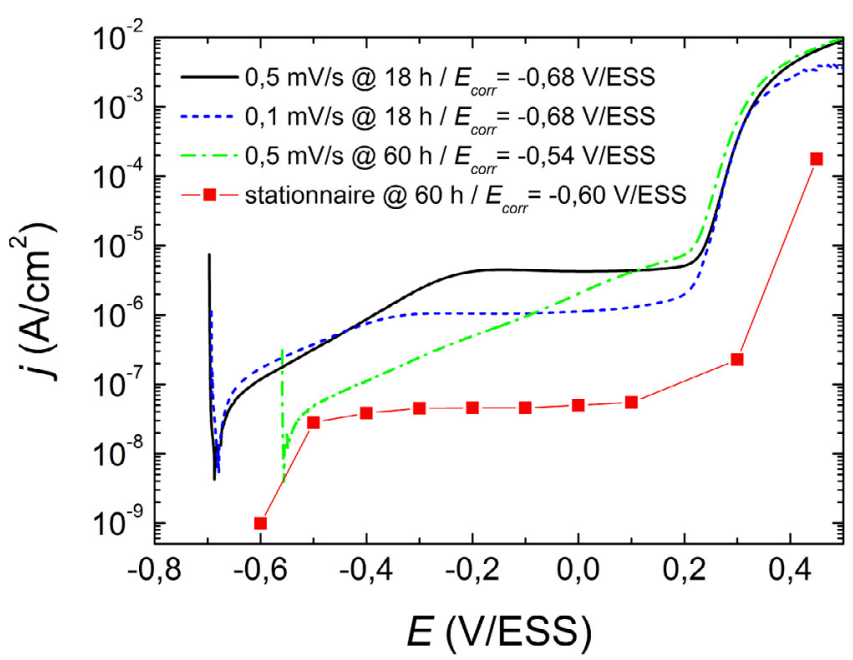

Fig. 3. Courbes de polarisation anodiques stationnaire et non stationnaires de l'acier $\mathrm{C} 15$ en solution $\mathrm{NaOH} 0,1 \mathrm{M}$, après 18 ou $60 \mathrm{~h}$ d'immersion au potentiel de corrosion. Les valeurs de $E_{\text {corr }}$ avant tracé sont indiquées sur la figure.

Fig. 3. Steady-state and non steady-state anodic polarization curves of $\mathrm{C} 15$ mild steel in $0.1 \mathrm{M} \mathrm{NaOH}$ solution, after 18 or $60 \mathrm{~h}$ of immersion at the corrosion potential. $E_{\text {corr }}$ values before plotting are indicated in the figure.

appelé limite basse fréquence de l'impédance ou encore résistance de polarisation, ne varie plus c'est-à-dire du temps à partir duquel les diagrammes sont superposés. Dans notre cas, la limite basse fréquence ne nous permet pas de déterminer la résistance de polarisation, mais étant donné que les diagrammes d'impédance se superposent à partir de $58 \mathrm{~h}$ d'immersion, le temps d'obtention d'un état quasi-stationnaire peut être estimé à une soixantaine d'heures environ.

\subsubsection{Courbes de polarisation anodiques}

La courbe de polarisation de l'acier C15 tracée après 18 h d'immersion à $E_{\text {corr }}$ dans la solution $\mathrm{NaOH} 0,1 \mathrm{M}$, avec une vitesse de balayage en potentiel de $0,5 \mathrm{mV} \cdot \mathrm{s}^{-1}$, est présentée à la Figure 3. Cette courbe présente un palier passif étendu sur plusieurs centaines de $\mathrm{mV}$, correspondant à une densité de courant de $4,2 \mu \mathrm{A} \cdot \mathrm{cm}^{-2}$. Le palier passif est suivi d'une augmentation de courant illustrant le domaine transpassif. Entre $E_{\text {corr }}$ et le potentiel de début de palier, une variation linéaire du courant peut être observée sur plusieurs centaines de $\mathrm{mV}$, mettant en évidence un comportement de type Tafel (réaction anodique totalement limitée par le transfert de charge).

La courbe anodique tracée après $18 \mathrm{~h}$ d'immersion à $E_{c o r r}$ dans la solution $\mathrm{NaOH} 0,1 \mathrm{M}$ et avec une vitesse de balayage de $0,1 \mathrm{mV} \cdot \mathrm{s}^{-1}$ est également présentée à la Figure 3. L'allure de cette courbe est similaire à celle de la courbe tracée à $0,5 \mathrm{mV} \cdot \mathrm{s}^{-1}$. Cependant, une diminution de la densité de courant passif $\left(1,0 \mu \mathrm{A} . \mathrm{cm}^{-2}\right)$ ainsi qu'un comportement de type Tafel dans une gamme plus étroite de potentiel peuvent être observés. 


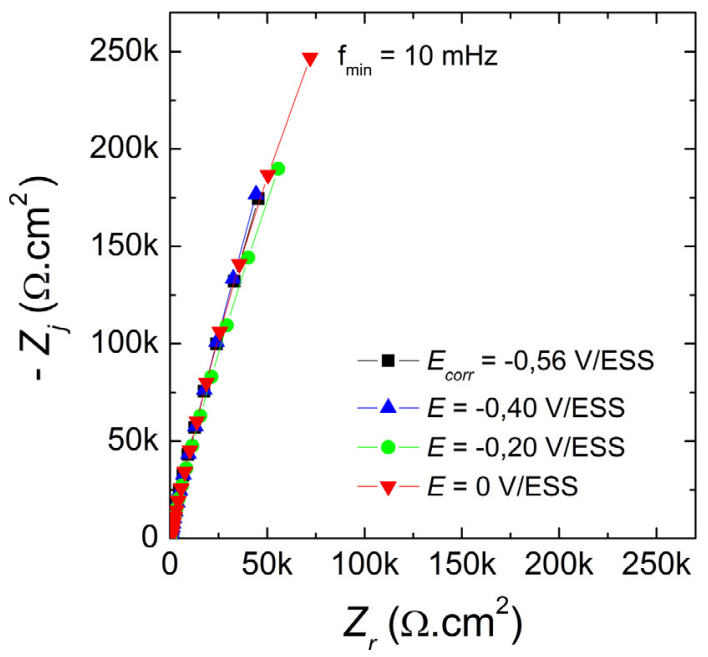

(a)

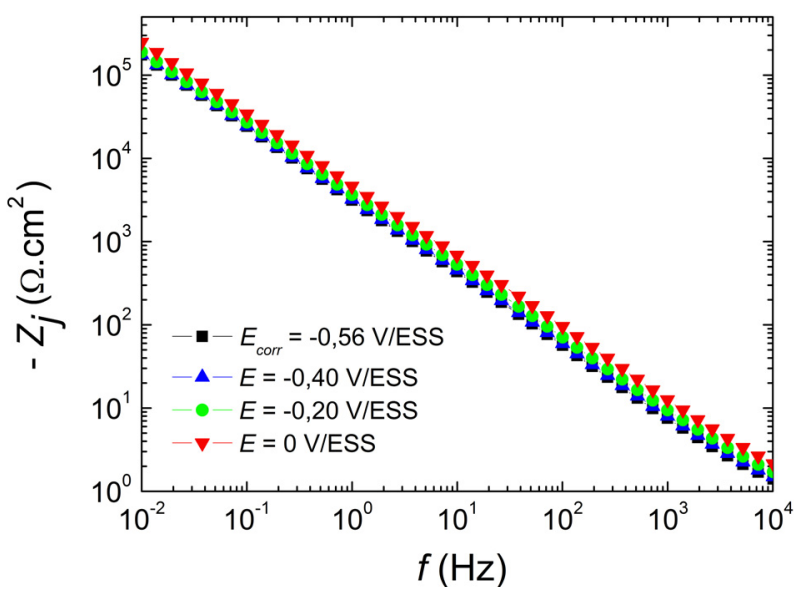

(b)

Fig. 4. Diagrammes d'impédance de l'acier $\mathrm{C} 15$ en solution $\mathrm{NaOH} 0,1 \mathrm{M}$, tracés à $E_{\text {corr }}$ après 60 h d'immersion et à différents potentiels anodiques. (a) Diagrammes de Nyquist, (b) partie imaginaire de l'impédance en fonction de la fréquence. Nombre de points par décade : 7 .

Fig. 4. Impedance data of $C 15$ mild steel in $0.1 \mathrm{M} \mathrm{NaOH}$ solution, plotted at $E_{\text {corr }}$ after $60 \mathrm{~h}$ of immersion and at different anodic potentials. (a) Nyquist plots and (b) imaginary part of the impedance as a function of frequency. 7 points per decade.

Étant donnée l'influence non négligeable de la vitesse de balayage sur les densités de courant anodiques et, en particulier, sur la densité de courant passif, il a été décidé de tracer une courbe de polarisation anodique stationnaire, c'est-à-dire point par point et après $60 \mathrm{~h}$ d'immersion à $E_{\text {corr }}$ (d'après les conclusions de la Sect. 3.1.1). La courbe stationnaire présentée sur la Figure 3 est tracée en maintenant à chaque point la polarisation jusqu'à l'obtention d'un courant dont la variation ne dépasse pas $0,4 \mu \mathrm{A} . \mathrm{cm}^{-2}$ en $2 \mathrm{~min}$ (valeur de la pente de la courbe courant $v s$. temps après $\sim 4 \mathrm{~h}$ de polarisation). Il a été montré que deux courbes stationnaires tracées dans les mêmes conditions se superposent, montrant ainsi la reproductibilité de telles mesures (résultats non présentés ici). Le comportement de type Tafel n'est plus observé en conditions quasi-stationnaires; par conséquent, la présence d'une portion linéaire des courbes tracées à $0,5 \mathrm{mV} . \mathrm{s}^{-1}$ et $0,1 \mathrm{mV} \cdot \mathrm{s}^{-1}$ s'explique par la non-stationnarité du système. De plus, la densité de courant passif stationnaire $j_{\text {stat }}$ est environ 100 fois plus faible que celle enregistrée à $0,5 \mathrm{mV} \cdot \mathrm{s}^{-1}\left(j_{\text {stat }} \sim 5 \times 10^{-8} \mathrm{~A} \cdot \mathrm{cm}^{-2}\right)$.

Une courbe de polarisation anodique a également été tracée après $60 \mathrm{~h}$ d'immersion à $E_{\text {corr }}$ mais avec une vitesse de balayage de $0,5 \mathrm{mV} \cdot \mathrm{s}^{-1}$ (Fig. 3). Cette courbe présente un comportement de type Tafel dans un très large domaine de potentiel et un palier passif très étroit, correspondant à une densité de courant passif similaire à celle mesurée à la même vitesse de balayage après $18 \mathrm{~h}$ d'immersion à $E_{\text {corr }}$. Cette portion linéaire observée sur la quasi-totalité du domaine passif illustre à nouveau la non-stationnarité du système. Ce résultat confirme que pour ce système, la courbe de polarisation anodique stationnaire doit être tracée point par point et après $60 \mathrm{~h}$ d'immersion à $E_{\text {corr }}$.
Une autre conclusion tirée de ces résultats est que le palier passif peut être extrapolé jusqu'à $E_{c o r r}$, c'est-àdire que la densité de courant de corrosion $j_{\text {corr }}$ peut être estimée égale à $j_{\text {stat }}[14]$.

\subsubsection{Diagrammes d'impédance électrochimique anodiques}

Afin de confirmer les conclusions tirées des courbes de polarisation anodiques, des diagrammes d'impédance électrochimique sont tracés au potentiel de corrosion $\left(E_{\text {corr }}=-0,56 \mathrm{~V} / \mathrm{ESS}\right)$ après $60 \mathrm{~h}$ d'immersion ainsi qu'à différents potentiels anodiques en conditions stationnaires (mesures réalisées lors de l'obtention des courbes de polarisation anodiques stationnaires présentées sur la Fig. 3). Ces différents diagrammes sont tracés dans le plan complexe (diagrammes de Nyquist) sur la Figure 4a. Ils ont la même allure quel que soit le potentiel et présentent une boucle capacitive de large diamètre caractéristique d'une couche passive. Tous les diagrammes se superposent mais présentent un glissement en fréquence lorsque le potentiel devient plus anodique. Ces résultats montrent que le comportement électrochimique de l'acier est similaire à $E_{c o r r}$ et à un potentiel anodique appartenant au domaine passif et que ce dernier s'étend au minimum de $E_{c o r r}$ jusqu'à $0 \mathrm{~V} / \mathrm{ESS}$.

La représentation de la partie imaginaire de l'impédance en fonction de la fréquence en coordonnées logarithmiques (Fig. 4b) est une droite de pente inférieure à 1 en valeur absolue, mettant ainsi en évidence un comportement CPE (Constant-Phase Element, en anglais). L'impédance d'un CPE est donnée par :

$$
Z_{\mathrm{CPE}}=\frac{1}{Q(j \omega)^{\alpha}}
$$


Tableau 2. Paramètres $\alpha$ et $Q$ du CPE estimés graphiquement à partir des données d'impédance de la Figure 4, épaisseur de la couche d'oxyde $\delta$ (Eq. (5)) et capacité de la couche d'oxyde $C_{o x}$ (Eq. (6)) à $E_{\text {corr }}$ et à différents potentiels anodiques.

Table 2. CPE parameters $\alpha$ and $Q$ graphically obtained from impedance data presented in Figure 4, oxide layer thickness $\delta$ (Eq. (5)), and oxide layer capacitance $C_{o x}(E q .(6))$ at $E_{c o r r}$ and at different anodic potentials.

\begin{tabular}{ccccc}
\hline Potentiel (V/ESS $)$ & $\alpha$ & $Q\left(\Omega^{-1} \cdot \mathrm{cm}^{-2} \cdot \mathrm{s}^{\alpha}\right)$ & $\delta(\mathrm{nm})$ & $C_{\text {ox }}\left(\mathrm{F} . \mathrm{cm}^{-2}\right)$ \\
\hline$E_{\text {corr }}=-0,56$ & 0,87 & $6,28 \times 10^{-5}$ & 3,0 & $3,5 \times 10^{-6}$ \\
$-0,40$ & 0,86 & $6,29 \times 10^{-5}$ & 3,3 & $3,2 \times 10^{-6}$ \\
$-0,20$ & 0,86 & $5,41 \times 10^{-5}$ & 3,9 & $2,7 \times 10^{-6}$ \\
0 & 0,85 & $4,35 \times 10^{-5}$ & 6,0 & $1,7 \times 10^{-6}$ \\
\hline
\end{tabular}

Elle fait apparaître deux paramètres, $Q$ et $\alpha$, indépendants de la fréquence. Quand $\alpha=1, Q$ a les dimensions d'une capacité, i.e. F.cm ${ }^{-2}$; quand $0<\alpha<1, Q$ est exprimé en $\mathrm{s}^{\alpha} \cdot \Omega^{-1} \cdot \mathrm{cm}^{-2}$ ou encore $\mathrm{F} . \mathrm{S}^{(\alpha-1)} \cdot \mathrm{cm}^{-2}$ et $\alpha$ est adimensionnel.

Un comportement CPE peut être attribué à une distribution de constantes de temps soit le long de la surface de l'électrode (distribution surfacique) soit le long de l'axe normal à la surface d'électrode (distribution normale). Une distribution surfacique peut provenir d'hétérogénéités de surface (joints de grains, faces cristallines...) ou de distributions non uniformes de courant et de potentiel induites par la géométrie d'électrode. Tandis qu'une distribution normale peut être engendrée par une électrode poreuse ou une variation de conductivité au sein de couches de surface telles que les films d'oxyde [15].

Dans le cas d'un circuit R//CPE, les paramètres du CPE $\alpha$ et $Q$ peuvent être estimés graphiquement selon la méthode présentée par Orazem et al. [16]. $\alpha$ correspond à la valeur absolue de la pente de la courbe $\log \left|Z_{j}\right|$ vs. $\log f$ :

$$
\alpha=\left|\frac{d \log \left|Z_{j}(f)\right|}{d \log f}\right|
$$

et $Q$ est obtenu à partir de $\alpha$ comme suit :

$$
Q=-\frac{1}{Z_{j}(f)(2 \pi f)^{\alpha}} \times \sin \left(\frac{\alpha \pi}{2}\right)
$$

Les valeurs estimées pour $\alpha$ et $Q$ sont présentées dans le Tableau 2.

Les valeurs estimées pour $\alpha$ et $Q$ correspondent à celles habituellement rencontrées pour des couches d'oxyde de type couches passives [17]. Dans le cas d'une couche d'oxyde, le comportement CPE est généralement attribué à une distribution normale de constantes de temps induite par une variation significative de résistivité au sein du film et le modèle loi puissance doit être utilisé afin d'analyser les données d'impédance [18]. Ce modèle suppose la distribution de résistivité suivante :

$$
\frac{\rho}{\rho_{\delta}}=\left(\frac{\rho_{\delta}}{\rho_{0}}+\left(1-\frac{\rho_{\delta}}{\rho_{0}}\right) \xi^{\gamma}\right)^{-1}
$$

où $\xi$ est la position adimensionnelle au sein de la couche d'oxyde $\left(\xi=y / \delta\right.$ avec $\delta$ l'épaisseur de la couche), $\rho_{0}$ et $\rho_{\delta}$ sont les valeurs limites de résistivité aux interfaces (à $\xi=0$ et $\xi=\delta$, respectivement), et $\gamma$ est une constante indiquant le degré de variation de la résistivité.
En supposant que la constante diélectrique $\varepsilon$ est indépendante de la position, il a été montré que $\alpha=$ $(\gamma-1) / \gamma$ et l'équation reliant les paramètres CPE aux propriétés physiques du film est la suivante :

$$
Q=\frac{\left(\varepsilon \varepsilon_{0}\right)^{\alpha}}{g \delta \rho_{\delta}^{1-\alpha}}
$$

où $\varepsilon_{0}$ est la permittivité du vide et $g$ est une fonction de $\alpha\left(g=1+2,88(1-\alpha)^{2,375}\right)[18]$.

Ce modèle loi puissance est utilisé dans ce travail pour analyser les données d'impédance de l'acier C15 en solution $\mathrm{NaOH}$ 0,1 M à différents potentiels. L'épaisseur de la couche d'oxyde est calculée par application de l'équation (5) avec les valeurs de $\alpha$ et $Q$ estimées graphiquement à partir des données d'impédance (Tab. 1), $\varepsilon=$ 12 (constante diélectrique de $\mathrm{Fe}_{2} \mathrm{O}_{3}$ [17]), $\varepsilon_{0}=8,8542 \times$ $10^{-14} \mathrm{~F} . \mathrm{cm}^{-1}$ et $\rho_{\delta}=500 \Omega . \mathrm{cm}$ (valeur déterminée pour un acier inoxydable $\mathrm{Fe}-17 \mathrm{Cr}$ en solution $\mathrm{Na}_{2} \mathrm{SO}_{4}$ 0,05 M désaérée à $\mathrm{pH} 4$ [17]).

À partir de l'épaisseur de la couche d'oxyde, la capacité de cette couche $C_{o x}$ peut être calculée comme suit $[17]$ :

$$
C_{o x}=\frac{\varepsilon \varepsilon_{0}}{\delta}
$$

Les valeurs calculées pour $\delta$ et de $C_{o x}$ sont rassemblées dans le Tableau 2. L'épaisseur de la couche d'oxyde est de l'ordre de quelques nanomètres, ce qui est en accord avec les valeurs habituellement trouvées pour les couches passives formées sur matériaux ferreux [19]. Ce résultat sera confirmé par les analyses XPS présentées dans la suite de ce travail. De plus, les valeurs de $Q$ et de $C_{O x}$ diminuent lorsque le potentiel anodique augmente, en accord avec l'augmentation de l'épaisseur de la couche d'oxyde $\delta$ (Tab. 2). Cet épaississement du film passif avec le potentiel a déjà été observé par XPS par Maurice et al. pour un acier inoxydable Fe-22Cr en milieu acide [20]. Par ailleurs, les résultats XPS obtenus par Haupt et al. avec du fer et des alliages $\mathrm{Fe}-\mathrm{Cr}$ en milieu alcalin montrent un comportement linéaire entre l'épaisseur des films passifs formés et le potentiel appliqué [21].

Ces résultats confirment que le comportement CPE observé à $E_{c o r r}$ et à potentiel anodique provient d'une distribution normale de résistivité au sein du film passif.

\subsubsection{Analyse de surface par XPS}

Les analyses de surface par XPS de l'acier doux C15 ont été réalisées après $60 \mathrm{~h}$ d'immersion dans la solution 


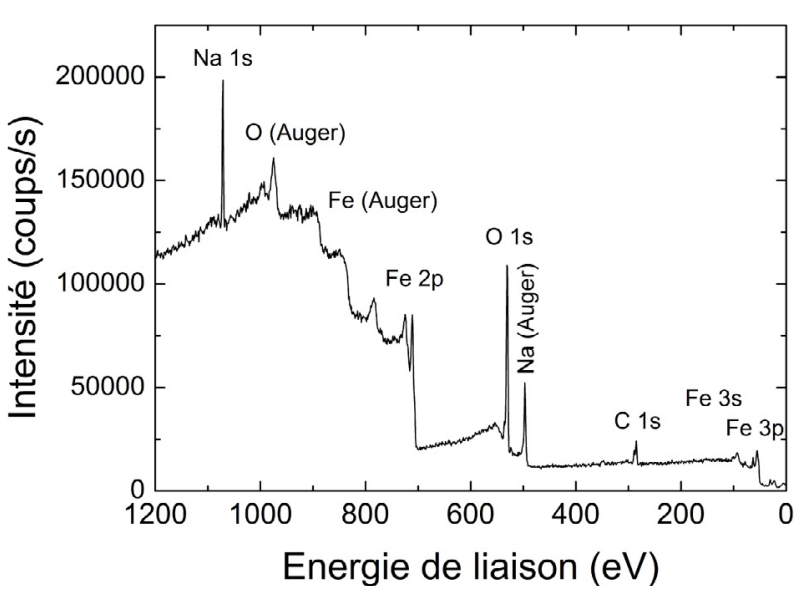

(a)

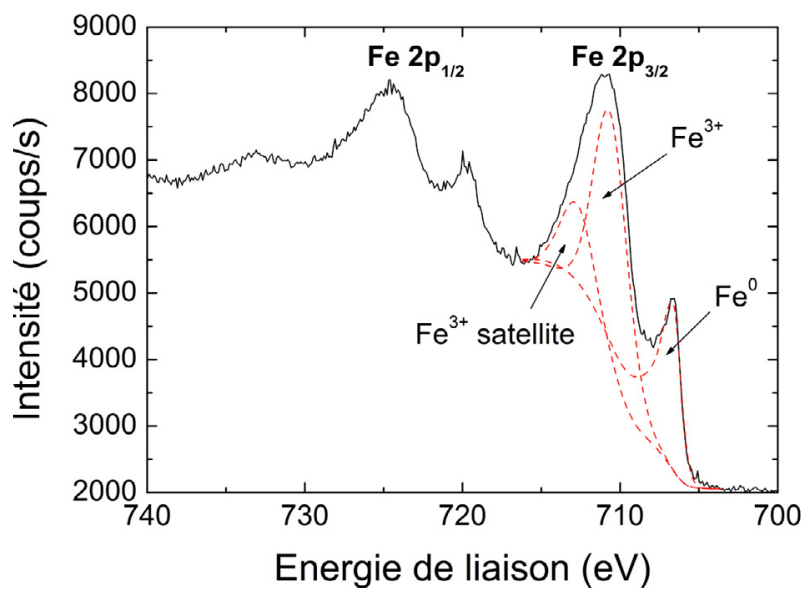

(b)

Fig. 5. Spectres XPS (a) général et (b) du niveau de cour Fe $2 p$ de l'acier $\mathrm{C} 15$ après 60 h d'immersion à $E_{c o r r}$ en solution $\mathrm{NaOH} 0,1 \mathrm{M} .(-)$ Spectres expérimentaux et $(---)$ décomposition.

Fig. 5. X-ray photoelectron spectroscopy (XPS) (a) general spectra and (b) Fe 2p core level spectra of C15 mild steel after $60 \mathrm{~h}$ of immersion at $E_{\text {corr }}$ in $0.1 \mathrm{M} \mathrm{NaOH}$ solution. (_-) Experimental spectra and (- - -) peak decomposition.

$\mathrm{NaOH}$ 0,1 M afin d'estimer la composition chimique et l'épaisseur de la couche d'oxyde formée.

Dans un premier temps, l'enregistrement du spectre général (Fig. 5a), balayant l'ensemble des énergies de liaison entre 0 et $1200 \mathrm{eV}$, permet d'identifier les différents éléments présents sur la surface de l'électrode. Dans notre cas, ce spectre indique la présence de fer (éléments majoritaires composant l'acier doux), de sodium provenant de la solution d'étude, ainsi que d'oxygène (oxyde, eau adsorbée... ) et de carbone.

La décomposition du spectre du niveau de cour $\mathrm{Fe}$ $2 p_{3 / 2}$ en contributions élémentaires met en évidence trois pics (Fig. 5b). Le premier pic, centré sur une énergie de liaison de 706,5 eV, est caractéristique du fer à l'état métallique $\left(\mathrm{Fe}^{0}\right)$. Les deuxième et troisième contributions à 710,2 et $712,1 \mathrm{eV}$ correspondent au pic principal et au pic satellite, respectivement, du fer au degré d'oxydation + III $\left(\mathrm{Fe}^{3+}\right.$ dans $\mathrm{Fe}_{2} \mathrm{O}_{3}$ et/ou FeOOH). Aucun épaulement significatif n'est relevé pour des énergies de liaisons voisines de $709 \mathrm{eV}$, caractéristiques du fer au degré d'oxydation +II [22].

Le rapport d'intensité $I_{\mathrm{Fe}}^{\text {métal }} / I_{\mathrm{Fe}}^{\text {oxyde }}$, avec $I_{\mathrm{Fe}}^{\text {oxyde }}$ égal à l'aire sous les pics principal et satellite du fer oxydé, permet de remonter à l'épaisseur de la couche d'oxyde formée sur la surface de l'échantillon. Après $60 \mathrm{~h}$ d'immersion à $E_{c o r r}$ dans la solution de référence $\mathrm{NaOH} 0,1 \mathrm{M}$, l'épaisseur calculée est de 2,4 $\pm 0,3 \mathrm{~nm}$. Cette valeur est en très bon accord avec celle estimée à partir des données d'impédance à $E_{\text {corr }}(3,0 \mathrm{~nm}$; Tab. 2).

\subsection{Solutions interstitielles synthétiques}

\subsubsection{Mesures électrochimiques}

Les courbes de polarisation anodiques stationnaires et les diagrammes d'impédance électrochimique
Tableau 3. Potentiel de corrosion $E_{\text {corr }}$ après 60 h d'immersion et densité de courant passif stationnaire $j_{\text {stat }}$ dans $\mathrm{NaOH}$ 0,1 M et les différentes solutions interstitielles synthétiques.

Table 3. Corrosion potential $E_{\text {corr }}$ after $60 \mathrm{~h}$ of immersion and steady-state passive current density $j_{\text {stat }}$ in $0.1 \mathrm{M} \mathrm{NaOH}$ solution and different simulated concrete pore solutions.

\begin{tabular}{ccc}
\hline Solution & $E_{\text {corr }}(\mathrm{V} / \mathrm{ESS})$ & $j_{\text {stat }}\left(\mathrm{A} . \mathrm{cm}^{-2}\right)$ \\
\hline CEM I & $-0,81$ & $1,21 \times 10^{-6}$ \\
CEM II & $-0,77$ & $4,4 \times 10^{-7}$ \\
CEM III & $-0,65$ & $7,1 \times 10^{-8}$ \\
NaOH 0,1 M & $-0,60$ & $4,6 \times 10^{-8}$ \\
\hline
\end{tabular}

à $E_{c o r r}$, tracés dans les différentes solutions interstitielles synthétiques après $60 \mathrm{~h}$ d'immersion à $E_{\text {corr }}$, sont présentées sur la Figure 6 et sont comparées à celles obtenues dans $\mathrm{NaOH}$ 0,1 M. Les valeurs de $E_{\text {corr }}$ avant le tracé des courbes de polarisation de la Figure 6a sont données sur la Figure 6b ainsi que dans le Tableau 3.

L'allure de toutes les courbes de polarisation est similaire (Fig. 6a). Cependant, des différences de largeur de palier passif ainsi que de densité de courant passif peuvent être observées. Ainsi pour les trois solutions interstitielles synthétiques, le palier est d'autant plus large que $E_{\text {corr }}$ est cathodique (Tab. 3). Par ailleurs, la densité de courant passif stationnaire dépend de la solution d'étude. Les valeurs de $j_{\text {stat }}$ sont rassemblées dans le Tableau 3. Ainsi, $j_{\text {stat }}$ est 15 fois plus élevé dans la solution CEM I que dans la solution CEM III. Le classement du pouvoir passivant des différentes solutions, obtenu à partir des courbes de polarisation, est le suivant : CEM I $<$ CEM II $<$ CEM III $\leq \mathrm{NaOH}$. Ce classement fait apparaître une corrélation entre la concentration en ions sulfate de l'électrolyte (Tab. 1), le potentiel de corrosion et la densité de courant passif : plus la concentration en sulfate est importante, plus $E_{\text {corr }}$ est 


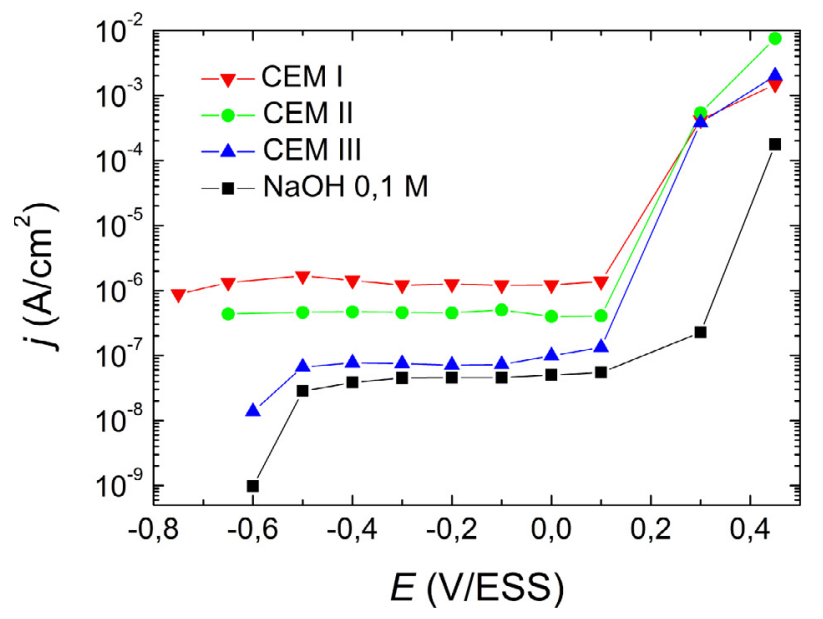

(a)

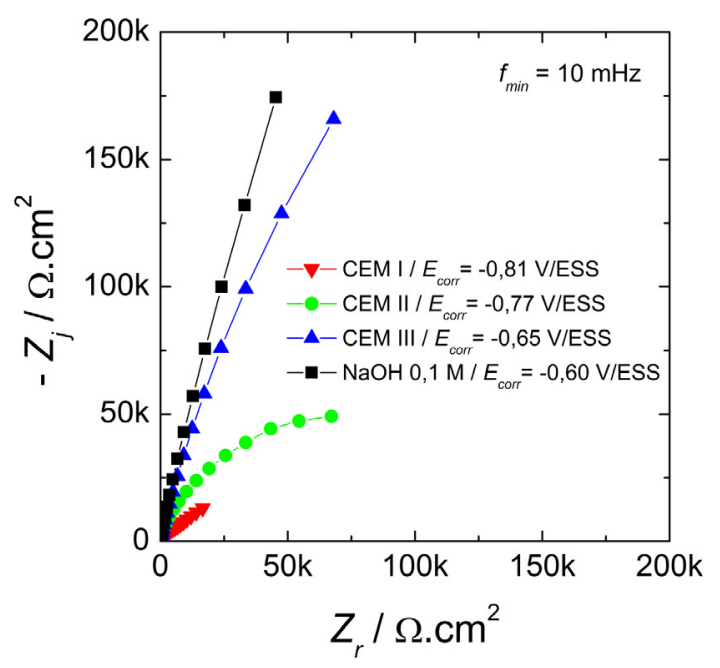

(b)

Fig. 6. (a) Courbes de polarisation anodiques stationnaires et (b) diagrammes d'impédance à $E_{c o r r}$ (représentation de Nyquist) de l'acier $\mathrm{C} 15$ après $60 \mathrm{~h}$ d'immersion à $E_{\text {corr }}$ dans $\mathrm{NaOH} \mathrm{0,1} \mathrm{M}$ et les différentes solutions interstitielles synthétiques. Le pH est ajusté à 13 avant chaque expérience.

Fig. 6. (a) Steady-state anodic polarization curves and (b) impedance diagrams at $E_{\text {corr }}$ (Nyquist plots) of C15 mild steel after $60 \mathrm{~h}$ of immersion at $E_{\text {corr }}$ in $0.1 \mathrm{M} \mathrm{NaOH}$ solution and different simulated concrete pore solutions. pH was adjusted to 13 before each experiment.

cathodique, plus la densité de courant passif stationnaire est élevée et moins bonne est la qualité de la couche d'oxyde. Ces résultats sont en accord avec ceux d'Abd El Haleem et al. qui ont mis en évidence l'effet néfaste des ions $\mathrm{SO}_{4}^{2-}$ sur le comportement à la corrosion d'un acier doux et, en particulier, la diminution du potentiel de corrosion accompagnant l'augmentation de la concentration en sulfate $[23,24]$. En effet, en milieu alcalin sulfaté, une compétition s'établit entre la croissance/cicatrisation du film d'oxyde par les ions $\mathrm{OH}^{-}$et sa destruction par les ions agressifs $\mathrm{SO}_{4}^{2-}$. Lorsque la concentration en sulfate est suffisamment élevée, le processus de destruction de la couche d'oxyde prédomine, pouvant ainsi induire la corrosion par piqûres du matériau. Par ailleurs, Ghods et al. ont montré que la présence de $\mathrm{SO}_{4}^{2-}$ dans la solution interstitielle avait un effet néfaste significatif sur les propriétés protectrices des films passifs formés sur les armatures métalliques [11].

De même qu'en solution $\mathrm{NaOH} \quad 0,1$ M, les diagrammes de Nyquist tracés dans les différentes solutions synthétiques présentent une boucle capacitive dont le diamètre est fonction de la solution étudiée. Plus la limite basse fréquence de l'impédance ou résistance de polarisation est élevée, plus la couche d'oxyde est protectrice et plus la solution est passivante. On obtient ainsi, à partir des données d'impédance, le même classement du pouvoir passivant des solutions que celui déduit des courbes de polarisation $(\mathrm{CEM} \mathrm{I}<\mathrm{CEM} \mathrm{II}<\mathrm{CEM} \mathrm{III} \leq \mathrm{NaOH})$.

Étant donné que le $\mathrm{pH}$ des différentes solutions est identique et égal à 13, l'ensemble des résultats électrochimiques permet de conclure que le pH n'est pas le seul paramètre déterminant pour prédire le comportement électrochimique de l'acier : à $\mathrm{pH}$ constant, $j_{\text {corr }}$ et
$E_{c o r r}$ dépendent de la composition de la solution utilisée. Cette conclusion est en accord avec les travaux de Ghods et al. [11].

\subsubsection{Analyse de surface par XPS}

La composition chimique et l'épaisseur de la couche d'oxyde ainsi que les espèces présentes à la surface de l'acier doux immergé pendant $60 \mathrm{~h}$ à $E_{\text {corr }}$ dans les différentes solutions synthétiques ont été déterminées par XPS. Sur les spectres généraux (Fig. 7a), on peut observer les pics du fer, du carbone et de l'oxygène, de même que ceux des éléments provenant de l'électrolyte, à savoir le sodium, le calcium, le potassium et le soufre. L'intensité de ces pics est différente selon le milieu d'étude. Les échantillons ont été rincés avec la solution dans laquelle ils ont été préalablement immergés, ce qui explique la présence assez marquée de sels $\left(\mathrm{Na}^{+}, \mathrm{Ca}^{2+}, \mathrm{K}^{+}\right.$et $\left.\mathrm{SO}_{4}^{2-}\right)$ sur la surface de l'acier.

Afin de déterminer les états d'oxydation du fer et leurs proportions relatives, les spectres du niveau de cour Fe $2 p_{3 / 2}$ sont décomposés en leurs différentes contributions. Dans le cas des échantillons immergés dans les solutions CEM I et CEM II (Figs. 7b et 7c), le pic Fe $2 p_{3 / 2}$ est décomposé en trois contributions attribuées au fer métallique $\left(\mathrm{Fe}^{0}\right)$ et au fer oxydé $\mathrm{Fe}^{3+}$ (pic principal et pic satellite). Pour la solution CEM III (Fig. 7d), la présence de fer au degré d'oxydation + II est clairement identifiée, avec un pic principal à $708,8 \mathrm{eV}$ et un pic satellite à $711,4 \mathrm{eV}$; en l'absence de ces deux contributions relatives à $\mathrm{Fe}^{2+}$, la décomposition du spectre du fer n'est pas satisfaisante. 
S. Chakri et al. : Matériaux \& Techniques 103, 209 (2015)

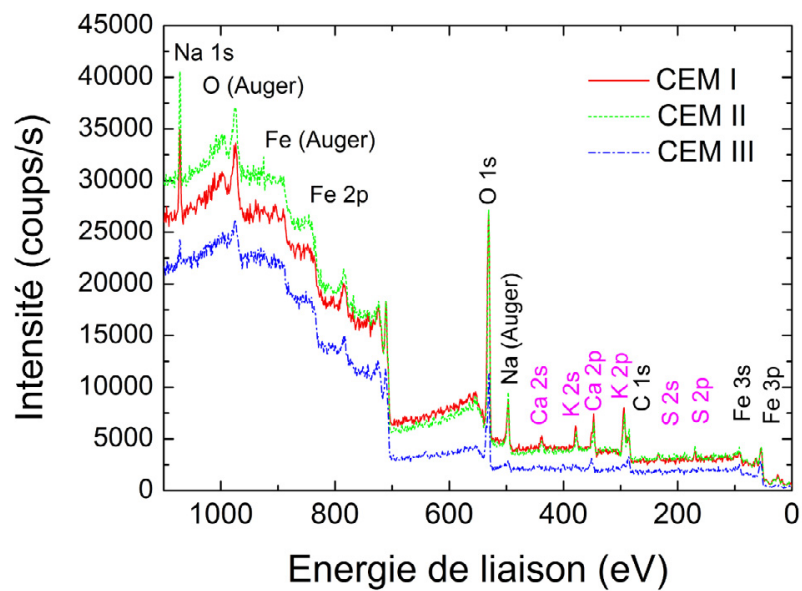

(a)

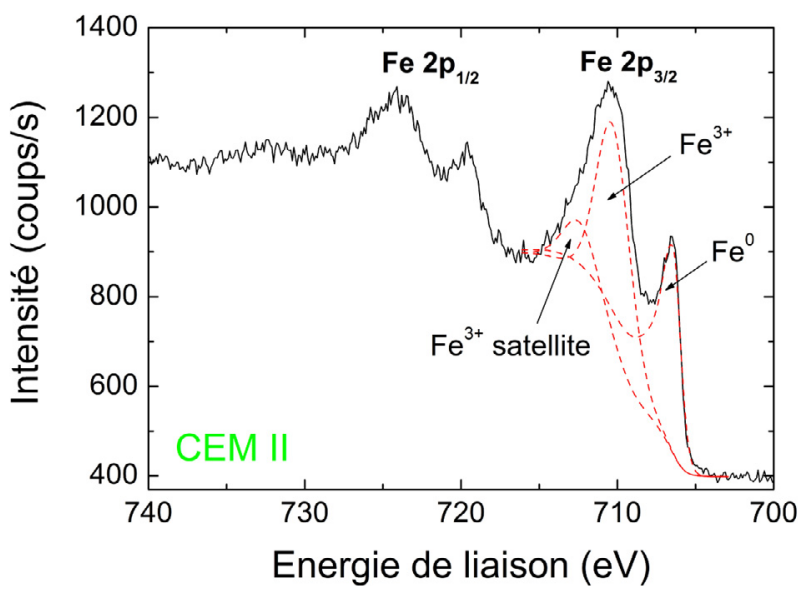

(c)

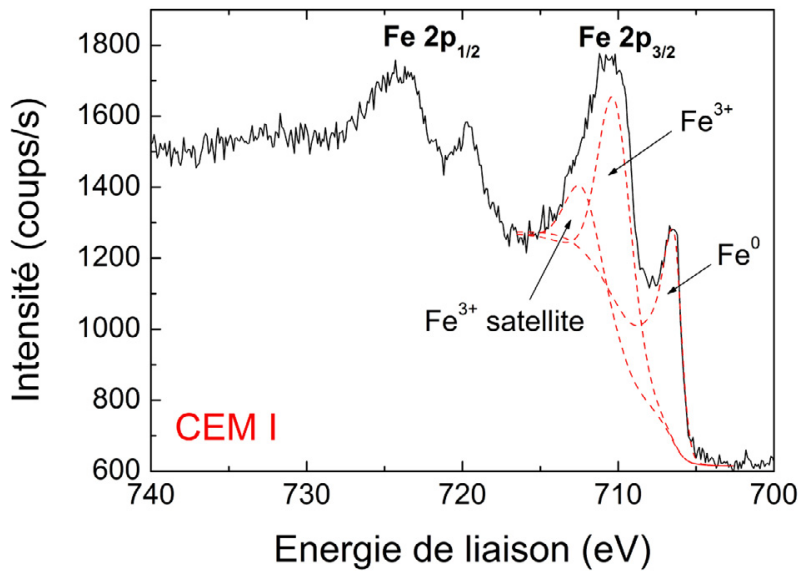

(b)

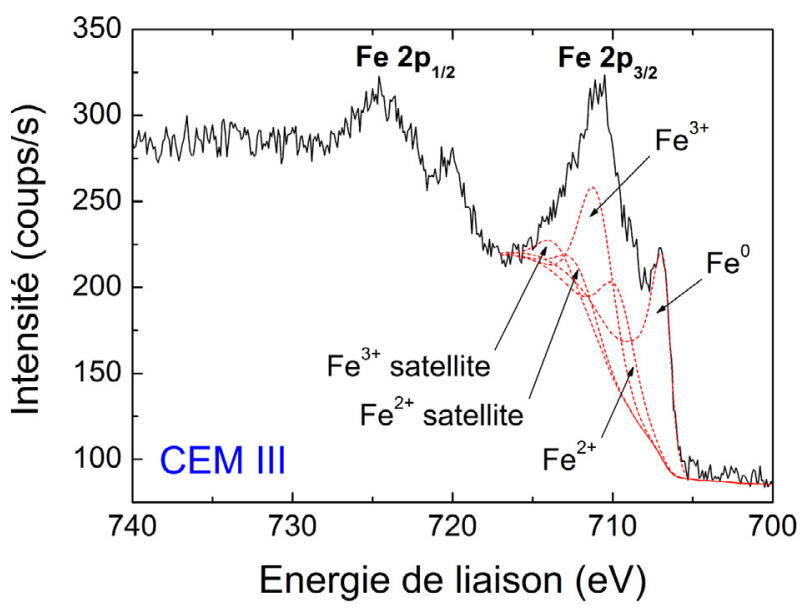

(d)

Fig. 7. Spectres XPS (a) généraux et du niveau de cœur Fe $2 p$ de l'acier $\mathrm{C} 15$ après $60 \mathrm{~h}$ d'immersion à $E_{\text {corr }}$ dans les solutions interstitielles synthétiques (b) CEM I, (c) CEM II et (d) CEM III. Niveau de cœur Fe $2 p_{3 / 2}$ : (—) spectres expérimentaux et $(---)$ décomposition.

Fig. 7. X-ray photoelectron spectroscopy (XPS) (a) general spectra and Fe 2p core level spectra of C15 mild steel after 60 h of immersion at $E_{\text {corr }}$ in (b) CEM I, (c) CEM II and (d) CEM III simulated concrete pore solutions. Fe 2p $p_{3 / 2}$ core level: (-) experimental spectra and $\left(-{ }_{-}\right)$peak decomposition.

La décomposition des spectres du fer permet de calculer l'épaisseur de la couche d'oxyde ainsi que les pourcentages atomiques de $\mathrm{Fe}^{3+}$ et de $\mathrm{Fe}^{2+}$ (Tab. 4). L'épaisseur de la couche d'oxyde est similaire quel que soit l'électrolyte et est comprise entre 2 et $3 \mathrm{~nm}$. En revanche, la composition de cette couche diffère selon le milieu. Ainsi, en solution CEM I et CEM II, elle est formée exclusivement de $\mathrm{Fe}^{3+}$, tandis qu'en solution CEM III, elle est constituée d'oxyde de $\mathrm{Fe}^{3+}$ et de $\mathrm{Fe}^{2+}$, avec un rapport $\mathrm{Fe}^{3+} / \mathrm{Fe}^{2+}$ de l'ordre de 4,5 .

Il a été montré que la présence de $\mathrm{Fe}^{2+}$ au voisinage de l'interface acier/oxyde rend la couche d'oxyde plus protectrice [6] et que lorsque la concentration en ions agressifs (e.g. $\mathrm{Cl}^{-}$) augmente, le rapport $\mathrm{Fe}^{2+} / \mathrm{Fe}^{3+}$ diminue conduisant ainsi à la dépassivation de l'acier [25]. Par conséquent, la présence de $\mathrm{Fe}^{2+}$ à la surface du matériau pourrait en partie expliquer le fort pouvoir passivant de
Tableau 4. Épaisseur et composition de la couche d'oxyde formée sur la surface d'acier C15, après $60 \mathrm{~h}$ d'immersion à $E_{\text {corr }}$ dans $\mathrm{NaOH} 0,1 \mathrm{M}$ et différentes solutions interstitielles synthétiques.

Table 4. Thickness and composition of the oxide layer formed on the $C 15$ mild steel surface after $60 \mathrm{~h}$ of immersion at $E_{\text {corr }}$ in $0.1 \mathrm{M} \mathrm{NaOH}$ solution and different simulated concrete pore solutions.

\begin{tabular}{cccc}
\hline Solution & $\begin{array}{c}\text { Épaisseur } \\
(\mathrm{nm})\end{array}$ & $\begin{array}{c}\mathrm{Fe}^{3+} \\
(\% \text { at. })\end{array}$ & $\begin{array}{c}\mathrm{Fe}^{2+} \\
(\% \text { at. })\end{array}$ \\
\hline CEM I & $2,7 \pm 0,3$ & 100 & 0 \\
CEM II & $2,3 \pm 0,3$ & 100 & 0 \\
CEM III & $2,9 \pm 0,3$ & 82 & 18 \\
NaOH 0,1 M & $2,4 \pm 0,3$ & 100 & 0 \\
\hline
\end{tabular}

la solution CEM III. Elle n'est cependant pas le seul élément clé permettant de comprendre la différence de 
comportement électrochimique de l'acier doux C15 dans les différents électrolytes, étant donné que le film passif développé dans $\mathrm{NaOH} 0,1 \mathrm{M}$ (solution la plus passivante) ne contient pas de $\mathrm{Fe}^{2+}$. Les mesures électrochimiques ont ainsi montré que la concentration en ions sulfate de l'électrolyte est un autre paramètre déterminant.

\section{Conclusions}

Cette étude montre l'influence de la composition chimique de la solution interstitielle de bétons jeunes (quelques minutes après gâchage du ciment) sur le caractère protecteur du film d'oxyde formé à la surface d'un acier doux C15 dans différentes solutions synthétiques, en combinant mesures électrochimiques et analyses de surface par XPS. Les résultats ainsi obtenus sont comparés à ceux obtenus dans la solution de référence $\mathrm{NaOH}$ 0,1 M, reproduisant l'environnement basique des armatures métalliques dans les bétons.

En milieu $\mathrm{NaOH} 0,1 \mathrm{M}$, les courbes de polarisation anodiques obtenues dans différentes conditions montrent l'intérêt des mesures électrochimiques stationnaires. Ainsi, une courbe de polarisation stationnaire doit être tracée, après $60 \mathrm{~h}$ d'immersion à $E_{c o r r}$, point par point et non pas en utilisant une vitesse de balayage même aussi faible que $0,1 \mathrm{mV} \cdot \mathrm{s}^{-1}$. Les résultats montrent que la densité de courant passif stationnaire $j_{\text {stat }}$ est environ 100 fois plus faible que celle enregistrée avec une vitesse de balayage de $0,5 \mathrm{mV} \cdot \mathrm{s}^{-1}$ et que le palier passif peut être extrapolé jusqu'au potentiel de corrosion. On en déduit que, la densité de courant de corrosion $j_{\text {corr }}$ est égale à la valeur de la densité de courant du palier passif $j_{\text {stat }}$. Les diagrammes d'impédance, tracés à $E_{c o r r}$ et dans le domaine anodique, mettent en évidence la présence d'une couche d'oxyde protectrice sur la surface de l'acier. L'épaisseur de cette couche, constituée uniquement de $\mathrm{Fe}^{3+}$, peut être déduite des données d'impédance à $E_{c o r r}$ ainsi que des données XPS; les valeurs obtenues par les deux techniques sont en très bon accord et de l'ordre de 2,5 à $3,0 \mathrm{~nm}$.

D'après les courbes de polarisation anodiques et les diagrammes d'impédance à $E_{\text {corr }}$ dans les trois solutions interstitielles synthétiques, il apparaît que le $\mathrm{pH}$ n'est pas le seul paramètre déterminant pour prédire le comportement électrochimique de l'acier. À pH 13 constant, $j_{\text {stat }}$ est 15 fois plus élevé dans la solution CEM I que dans la solution CEM III et le classement du pouvoir passivant des différentes solutions est le suivant : CEM I < CEM II < CEM III $\leq \mathrm{NaOH}$. Ce classement semble dépendre de la concentration en ions sulfate de l'électrolyte : plus les sulfates sont concentrés en solution, plus $j_{\text {stat }}$ est élevée et moins la solution est passivante. Les mesures électrochimiques montrent qu'un film passif de bonne qualité correspond à une faible densité de courant passif et à un potentiel de corrosion élevé. L'épaisseur de la couche d'oxyde estimée à partir des résultats XPS est similaire quel que soit l'électrolyte et est comprise entre 2 et $3 \mathrm{~nm}$. En revanche, la composition de cette couche diffère selon le milieu. Ainsi, en solution CEM I et CEM II, le fer est exclusivement sous la forme de $\mathrm{Fe}^{3+}$, tandis qu'en solution CEM III, la couche superficielle est constituée d'oxyde de $\mathrm{Fe}^{3+}$ et de $\mathrm{Fe}^{2+}$.

La concentration en ions sulfate (entrant dans la composition des solutions) ainsi que la présence ou l'absence de $\mathrm{Fe}^{2+}$ dans la couche d'oxyde sont des paramètres clé à prendre en compte pour comprendre la différence de comportement électrochimique de l'acier dans les différents électrolytes.

Remerciements. Les auteurs remercient l'Agence Nationale de la Recherche (ANR) pour le soutien financier du projet SEPOLBE (ANR-12-CDII-0004), ainsi que les pôles de compétitivité Energivie, Hydreos et Advancity qui ont parrainé ce travail.

\section{Références}

[1] H.A.F. Dehwah, M. Maslehuddin, S.A. Austin, Cem. Concr. Compos. 25 (2003) 513-525

[2] S.M. Abd El Haleem, E.E. Abd El Aal, S. Abd El Wanees, A. Diab, Corrosion Science 52 (2010) 3875-3882

[3] H. Oranowska, Z. Szklarskasmialowska, Corrosion Science 21 (1981) 735-747

[4] K.K. Sagoe-Crentsil, F.O. Glasser, Mag. Concrete Res. 41 (1989) 213-220

[5] M. Cohen, The passivity and breakdown of passivity on iron, in Passivity of Metals, edited by R.P. Frankenthal, J. Kurger, N.J. Princieton, The Electrochemical Society, 1978, p. 521

[6] H.B. Gunay, P. Ghods, O.B. Isgor, G.J.C. Carpenter, X. Wu, Appl. Surface Sci 274 (2013) 195-202

[7] Y.M. Tang, Y.F. Miao, Y. Zuo, G.D. Zhang, C.L. Wang, Constr. Buil. Mater. 30 (2012) 252-256

[8] G. Blanco, A. Bautista, H. Takenouti, Cem. Concr. Compos. 28 (2006) 212-219

[9] C. Monticelli, A. Frignani, G. Trabanelli, Cem. Concr. Res. 30 (2000) 635-642

[10] M. Saremi, E. Mahallati, Cem. Concr. Res. 32 (2002) 1915-1921

[11] P. Ghods, O.B. Isgor, G. McRae, T. Miller, Cem. Concr. Compos. 31 (2009) 2-11

[12] D. Rothstein, J.J. Thomas, B.J. Christensen, H.A. Jennings, Cem. Concr. Res. 32 (2002) 1663-1671

[13] A. Carnot, Mécanismes de corrosion des aciers constitutifs des moules pour les bétons, en présence d'un agent de démoulage, thèse de l'Université Paris 6, 2003

[14] A. Carnot, I. Frateur, P. Marcus, B. Tribollet, J. Appl. Electrochem. 32 (2002) 865-869

[15] V.M.-W. Huang, V. Vivier, M.E. Orazem, N. Pebere, B. Tribollet, J. Electrochem. Soc. 154 (2007) C81-C88

[16] M.E. Orazem, N. Pébère, B. Tribollet, J. Electrochem. Soc. 153 (2006) B129-B136

[17] B. Hirschorn, M.E. Orazem, B. Tribollet, V. Vivier, I. Frateur, M. Musiani, J. Electrochem. Soc. 157 (2010) C458-C463

[18] B. Hirschorn, M.E. Orazem, B. Tribollet, V. Vivier, I. Frateur, M. Musiani, J. Electrochem. Soc. 157 (2010) C452-C457 
S. Chakri et al. : Matériaux \& Techniques 103, 209 (2015)

[19] I. Frateur, L. Lartundo-Rojas, C. Methivier, A. Galtayries, P. Marcus, Electrochim. Acta 51 (2006) 15501557

[20] M. Maurice, W.P. Yang, P. Marcus, J. Electrochem. Soc. 143 (1996) 1182-1200

[21] S. Haupt, C. Calinski, U. Collisi, H.W. Hoppe, H.D. Speckmann, H.H. Strehblow, Surf. Interface Anal. 357 (1986) 9
[22] W.P. Yang, D. Costa, P. Marcus, J. Electrochem. Soc. 141 (1994) 2669-2676

[23] S.M. Abd El Haleem, S. Abd El Wanees, E.E. Abd El Aal, A. Diab, Corros. Sci. 52 (2010) 292-302

[24] S.M. Abd El Haleem, S. Abd El Wanees, A. Bahgat, Corros. Sci. 75 (2013) 1-15

[25] P. Ghods, O.B. Isgor, F. Bensebaa, D. Kingston, Corros. Sci. 58 (2012) 59-167 